\title{
Aspectos etológicos no suprimento de água em bovinos leiteiros
}

\author{
Dayane Lemos Teixeira* \\ Maria José Hötzel \\ Luiz Carlos Pinheiro Machado Filho \\ Daniel Enriquez Hidalgo \\ José Daniel Cazale
Laboratório de Etologia Aplicada, Departamento de Zootecnia e Desenvolvimento Rural Universidade Federal de Santa Catarina, Rodovia Admar Gonzaga, 1346 CEP 88.034-001, Florianópolis - SC, Brasil
*Autor para correspondência
dadaylt@hotmail.com

\section{Resumo}

Com o intuito de avaliar três alternativas adequadas de oferta de água para vacas leiteiras, alimentadas a base de pasto, na região sul do Brasil, foram disponibilizados três tipos de bebedouros, sendo: (1) formato circular, com $60 \mathrm{~cm}$ de altura x $120 \mathrm{~cm}$ de diâmetro e capacidade para 500L (500C); (2) formato circular, com $60 \mathrm{~cm}$ de altura x $60 \mathrm{~cm}$ de diâmetro e capacidade para $125 \mathrm{~L}(125 \mathrm{C})$ e (3) formato retangular, com $30 \mathrm{~cm}$ de altura x $100 \mathrm{~cm}$ de comprimento x $30 \mathrm{~cm}$ de largura e capacidade para 100L (100R). Os resultados obtidos através de testes individuais de preferência por parte das 17 vacas em lactação, mostraram $67 \%$ de acessos no bebedouro $500 \mathrm{C}, 18 \%$ no $100 \mathrm{R}$ e $15 \%$ no $125 \mathrm{C}$ ( $\mathrm{p}<0,001$ ). O bebedouro $500 \mathrm{C}$ em relação aos outros dois, apresentou maior volume de água ingerida $(\mathrm{p}<0,001)$, maior número de goles $(\mathrm{p}<0,001)$ e maior permanência de tempo bebendo $(\mathrm{p}<0,001)$. Quando os bebedouros $125 \mathrm{C}$ e $100 \mathrm{R}$ foram comparados, não houve diferença na preferência das vacas. Considerando os reconhecidos efeitos da ingestão de água na produção leiteira de vacas, conclui-se que o bebedouro escolhido pelas vacas deve ser recomendado.

Unitermos: bem-estar animal, comportamento animal, comportamento de beber, etologia, produção leiteira

\section{Abstract}

Ethological aspects on water supply for dairy cattle. In order to identify appropriate alternatives for the provision of water to dairy cattle, the preference of dairy cows among three types of water troughs used in pasturebased systems was evaluated. The first water trough was round, had a diameter of $120 \mathrm{~cm}$, was $60 \mathrm{~cm}$ high and held $500 \mathrm{~L}(500 \mathrm{C})$; the second was a round water trough, $60 \mathrm{~cm}$ in diameter, $60 \mathrm{~cm}$ high, and held $125 \mathrm{~L}(125 \mathrm{C})$; the last trough was rectangular, $30 \mathrm{~cm}$ high and $100 \mathrm{~cm}$ long, and held $100 \mathrm{~L}(100 \mathrm{R})$. Individual preference tests were carried out with 17 dairy cows. The cows preferred to drink $67 \%$ of the time from the $500 \mathrm{C}$ trough, $18 \%$ of the time from the $100 \mathrm{R}$ trough, and $15 \%$ of the time from the $125 \mathrm{C}$ trough $(\mathrm{p}<0.001)$. Also, these animals drank more water $(\mathrm{p}<0.001)$, took more sips $(\mathrm{p}<0.001)$ and spent more time drinking $(\mathrm{p}<0.001)$ from the 500C trough 
than from the other two troughs. When the 125C and 100R troughs were compared, no differences in the cows' preferences were found. Considering the known effects of water consumption on dairy cow milk production, it was concluded that the water trough preferred by the cows should be recommended.

Key words: animal behavior, animal welfare, drinking behavior, ethology, milk production

\section{Introdução}

Em aguadas naturais, como açudes, córregos e riachos, é comum os animais defecarem e urinaremna água, reduzindo a palatabilidade desta e, consequentemente, o seu consumo e o ganho de peso dos animais (Willms et al., 2002). Vários estudos mostraram a preferência de bovinos por ingerir água em bebedouros, quando dada a opção de escolha entre um bebedouro e uma aguada natural (Miner et al., 1992; Sheffield et al., 1997; Bica, 2005). Trabalhando com novilhos de corte, Bica (2005) encontrou que, além de preferirem beber em bebedouros, os animais que tinham acesso à água em bebedouros apresentaram maior ganho de peso do que aqueles que somente podiam beber água em um açude. Essas razões tornam o uso de bebedouros uma importante prática de manejo na criação de bovinos. Além disso, o uso de bebedouros pode diminuir o desgaste energético dos animais na busca por um ponto de água (Kamphues, 2000) e evitar a erosão causada pelos animais nas margens das aguadas naturais (Bagshaw et al., 2008).

Aspectos do suprimento de água em bebedouros, como a pressão, o fluxo (Andersson et al., 1984) e a localização do bebedouro - no corredor ou dentro do piquete (Coimbra et al., 2007) - influenciam a ingestão de água desses animais. O tamanho do bebedouro também afeta a preferência quanto o consumo de água de vacas leiteiras (Machado Filho et al., 2004). Nesse estudo, as vacas mostraram preferência e beberam mais água de bebedouros maiores do que nos menores. Posteriormente, Teixeira et al. (2006) mostraram que a área do espelho d'água é a característica que mais influencia a escolha do bebedouro em vacas leiteiras.

Para assegurar um consumo ótimo para cada animal, em sistemas de pastoreio rotativo, é necessário disponibilizar água dentro do potreiro, evitando assim que ocorra uma restrição no consumo relacionado ao comportamento social dos animais (Coimbra et al., 2007).
Isso requer a instalação de pelo menos um bebedouro para cada quatro potreiros (Pinheiro Machado, 2004). Muitos bebedouros encontrados em propriedades que manejam os animais em sistemas de pastoreio rotativo são feitos a partir de materiais reciclados ou de baixo custo, como tonéis utilizados para armazenar alimentos, caixas d'água de cimento ou até pneus. A escolha desses bebedouros é feita no intuito de reduzir o custo de instalação com equipamentos. Entretanto, considerando o efeito do consumo de água na produtividade leiteira (Burgos et al., 2001; NRC, 2001), a médio e longo prazo essas escolhas podem trazer prejuízos econômicos, se esses bebedouros restringirem o consumo de água dos animais.

O objetivo desta pesquisa foi avaliar a preferência de vacas leiteiras em três tipos de bebedouros de dimensões distintas, frequentemente recomendados por técnicos e utilizados na prática em projetos de Pastoreio Racional Voisin na Região Sul do Brasil.

\section{Materiais e Métodos}

$\mathrm{O}$ estudo foi conduzido na Unidade de Produção de Leite do Colégio Agrícola de Camboriú (CAC), da Universidade Federal de Santa Catarina (UFSC), localizado no município de Camboriú, Santa Catarina. $\mathrm{O}$ estudo foi realizado entre os meses de novembro de 2003 a janeiro de 2004.

O rebanho, composto por 17 vacas das raças Holandês e Jersey, era manejado no sistema de Pastoreio Racional Voisin (PRV). A média da produção leiteira das vacas durante o período do estudo foi de $13,5 \pm 2,1 \mathrm{~kg}$ de leite/vaca/dia, variando entre 4,5 e 31,7kg de leite/vaca/ dia. As vacas permaneciam todo o tempo em pastagem composta principalmente por brizantão (Brachiaria radicans), sendo levadas para a ordenha duas vezes ao dia, ocasião em que recebiam, como suplemento, capim-elefante (Pennisetum purpureum Schom.) fresco 
e picado, silagem de milho (Zea mays) e entre 1 e $4 \mathrm{~kg}$ de ração/vaca/dia, de acordo com o estádio de lactação.

Antes de iniciar o estudo, as vacas passaram por um período de 10 dias para habituação à rotina que foi implantada para a realização do experimento. Durante esses 10 dias de habituação, todos os bebedouros ficavam disponíveis no piquete dos testes de preferência (de aproximadamente $10 \mathrm{~m} \times 10 \mathrm{~m}$ ) e nos piquetes em que os animais estavam pastoreando, conforme rotação do sistema PRV.

Os animais eram recolhidos do piquete às 06h30min, para a realização da ordenha da manhã, que se estendia até às $08 \mathrm{~h} 30 \mathrm{~min}$. Em seguida, eram conduzidos até a pastagem, onde permaneciam até às $16 \mathrm{~h}$, quando eram recolhidos novamente para a ordenha da tarde. Os animais eram submetidos a uma restrição hídrica desde as $13 \mathrm{~h}$ e, logo após a ordenha, eram conduzidos individualmente até o piquete dos testes de preferência, onde os bebedouros estavam disponíveis. Os testes individuais de preferência das vacas tinham duração máxima de três minutos ou finalizavam quando a vaca parava de beber água e se dirigia para a porteira do piquete. Após o período de habituação, o bebedouro disponível nos piquetes do PRV era sempre diferente dos utilizados nos testes de preferência.

As 17 vacas foram submetidas a testes de preferência, com três tipos de bebedouros, de formatos e tamanhos diferentes (Figura 1):

- Bebedouro 500C: de Propileno de Alta Densidade (PEAD), circular, com $60 \mathrm{~cm}$ de altura x $120 \mathrm{~cm}$ de diâmetro e capacidade para 500L.

- Bebedouro 125C: de plástico, circular, com $60 \mathrm{~cm}$ de altura x $60 \mathrm{~cm}$ de diâmetro e capacidade para $125 \mathrm{~L}$;
- Bebedouro 100R: de plástico, retangular, com $30 \mathrm{~cm}$ de altura $\times 100 \mathrm{~cm}$ de comprimento $\times 30 \mathrm{~cm}$ de largura e capacidade para 100L.

Todos os bebedouros eram de cor azul. As três possibilidades de disposição dos bebedouros (direita, centro ou esquerda) foram sorteadas para cada dia, havendo três repetições para cada possibilidade, totalizando nove dias de testes.

Após a análise dos resultados dessa etapa experimental, o bebedouro considerado preferido pelas vacas foi retirado, sendo então conduzidos testes de preferência entre os outros dois bebedouros. As duas possibilidades diferentes de disposição dos bebedouros (esquerda ou direita) foram sorteadas para cada dia, havendo duas repetições para cada possibilidade, totalizando quatro dias de testes.

No piquete dos testes de preferência, os bebedouros estavam dispostos equidistantes à entrada do piquete $\mathrm{e}$ dois metros entre si. O observador estava posicionado em frente aos bebedouros e à vaca, distanciado destes em aproximadamente quatro metros. As medições feitas nestes testes foram:

- Volume consumido (L): antes da entrada de cada animal no piquete do teste de preferência, todos os bebedouros eram preenchidos com água até uma marca de referência na parede interna. Após a saída do animal do piquete, os bebedouros nos quais o animal tinha bebido água eram preenchidos com água até a marca de referência e, através de um hidrômetro (Tecnobrás ${ }^{\circledR}$, Brasil; precisão de 0,01L) acoplado na saída de água da mangueira, era possível medir com precisão a quantidade de água ingerida pelas vacas;

- Tempo bebendo (s): o tempo total que a boca do animal estava submersa, com o animal ingerindo água, foi registrado a partir de um cronômetro;
$500 \mathrm{C}$

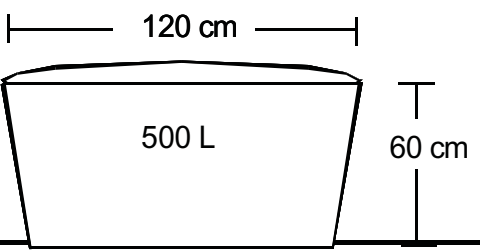

$125 C$

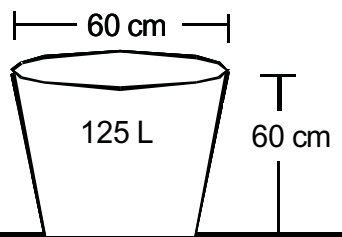

$100 R$

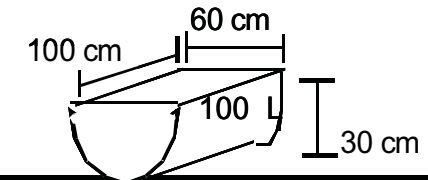

FIGURA 1: Bebedouros utilizados no experimento. 
- Número de goles (n): cada gole era considerado de acordo com o movimento da garganta do animal, denotando a deglutição da água, no momento que sua boca estava submersa. Os goles foram registrados com um contador manual;

- Taxa de ingestão (1/s): calculado pela razão do volume de água ingerido (L) pelo tempo total gasto ingerindo (s).

A temperatura da água de cada bebedouro foi medida antes, no meio e depois dos testes de preferência com todos os animais, através de termômetro $\left({ }^{\circ} \mathrm{C}\right.$; Delphin () ). A água dos bebedouros era trocada sempre que a diferença de temperatura superasse $3^{\circ} \mathrm{C}$.

\section{Análise estatística}

A unidade experimental foi cada animal, sendo utilizada a média das observações realizadas em cada vaca para cada teste. Os dados observados foram submetidos a uma análise da variância (Snedecor e Cochran, 1989). No modelo foram incluídos como fator fixo o bebedouro utilizado em cada teste de preferência. Para verificar alguma relação existente entre a preferência e a posição em que o mesmo estava disponível para as vacas (esquerda, centro ou direita; e esquerda ou direita), utilizou-se do teste do Chi-quadrado (Siegel, 1981). Os resultados são apresentados como média \pm erro padrão.

\section{Resultados e Discussão}

Quando os três bebedouros foram comparados, as vacas mostraram uma significativa preferência pelo bebedouro $500 \mathrm{C}$, bebendo $67 \%$ das vezes neste bebedouro, em comparação com $18 \%$ no bebedouro $100 \mathrm{R}$ e $15 \%$ no bebedouro $125 \mathrm{C}(\mathrm{p}<0,001)$. As vacas do presente experimento também consumiram mais água $(\mathrm{p}<0,0001)$, gastaram mais tempo bebendo $(\mathrm{p}<0,0001) \mathrm{e}$ deram maior número de goles $(\mathrm{p}<0,0001)$ no bebedouro $500 \mathrm{C}$ do que nos bebedouros 125C e 100R (Tabela 1). Os resultados encontrados nos testes de preferência corroboram os de Machado Filho et al. (2004) e Teixeira et al. (2006), que encontraram que as vacas preferem beber em bebedouros maiores. No experimento de
Machado Filho et al. (2004) as vacas beberam mais água, por mais tempo e deram maior número de goles no bebedouro mais alto $(60 \mathrm{~cm})$ e maior $(139 \mathrm{~cm} \times 95 \mathrm{~cm})$ que em outro mais baixo $(30 \mathrm{~cm})$ e menor $(126 \mathrm{~cm} \mathrm{x}$ $68 \mathrm{~cm}$ ). No experimento de Teixeira et al. (2006), estas mesmas variáveis avaliadas foram maiores no bebedouro de maior área superficial $\left(1,13 \mathrm{~m}^{2}\right)$ do que em outro de menor área superficial $\left(0,28 \mathrm{~m}^{2}\right)$.

Também houve uma diferença na taxa de ingestão entre os bebedouros $500 \mathrm{C}$ e $100 \mathrm{R}(\mathrm{p}<0,05)$, porém não houve diferença entre os bebedouros de $500 \mathrm{C}$ e $125 \mathrm{C}(\mathrm{p}<0,09)$. As vacas beberam $0,37 \pm 0,02 \mathrm{~L} / \mathrm{s}$ no bebedouro $500 \mathrm{C}, 0,28 \pm 0,04 \mathrm{~L} / \mathrm{s}$ no bebedouro $125 \mathrm{C}$ e $0,24 \pm 0,04 \mathrm{~L} / \mathrm{s}$ no bebedouro $100 \mathrm{R}$. A posição dos bebedouros (esquerda, centro ou direita) não influenciou a preferência das vacas.

TABELA 1: Consumo de água (L), tempo bebendo (s) e número de goles $(\mathrm{n})$ de vacas lactantes em diferentes bebedouros.

\begin{tabular}{lccc}
\multicolumn{1}{c}{ Testes } & $\begin{array}{c}\text { Volume con- } \\
\text { sumido (L) }\end{array}$ & $\begin{array}{c}\text { Tempo be- } \\
\text { bendo (s) }\end{array}$ & Goles (n) \\
\hline Três bebedouros & & & \\
500C & $14,56 \pm 1,48$ a & $39,47 \pm 4,31$ a & $25,12 \pm 2,98$ a \\
125C & $3,43 \pm 0,82 \mathrm{~b}$ & $9,44 \pm 2,34 \mathrm{~b}$ & $6,05 \pm 1,42 \mathrm{~b}$ \\
100R & $3,61 \pm 0,90 \mathrm{~b}$ & $10,82 \pm 2,76 \mathrm{~b}$ & $6,69 \pm 1,60 \mathrm{~b}$ \\
& $\mathrm{p}<0,0001$ & $\mathrm{p}<0,0001$ & $\mathrm{p}<0,0001$ \\
& & & \\
Dois bebedouros & & & \\
125C & $10,78 \pm 2,25$ & $29,34 \pm 6,21$ & $18,97 \pm 4,03$ \\
100R & $8,87 \pm 1,64$ & $28,75 \pm 5,25$ & $17,94 \pm 3,11$ \\
& $\mathrm{p}<0,5$ & $\mathrm{p}<0,9$ & $\mathrm{p}<0,8$ \\
\hline
\end{tabular}

* Letras diferentes indicam diferenças entre os tratamentos para uma determinada variável (Teste Exato de Fisher, $\mathrm{p}<0,05$ ).

Como no primeiro teste de preferência, comparando os três bebedouros, não foi encontrada diferença entre os bebedouros $125 \mathrm{C}$ e $100 \mathrm{R}$, outros testes foram realizados para verificar se as vacas mostrariam preferência caso apenas esses dois bebedouros, de menor volume e dimensões tão diferentes entre si, estivessem disponíveis. Entretanto, não foram encontradas diferenças em nenhuma das variáveis medidas (Tabela 1). A porcentagem do tempo gasto em cada bebedouro foi $50 \%$. As vacas beberam $0,61 \pm 0,48 \mathrm{~L} / \mathrm{s}$ no bebedouro $125 \mathrm{C}$ e $0,58 \pm 0,63 \mathrm{~L} / \mathrm{s}$ no bebedouro $100 \mathrm{R}$, não 
havendo diferença na taxa de ingestão de água entre os bebedouros $(p<0,8)$.

Na pesquisa realizada por Machado Filho et al. (2004), considerando que o bebedouro maior era mais largo, não foi possível concluir se a escolha das vacas foi baseada na área do espelho d'água do bebedouro ou na combinação da área do espelho d'água e altura. Contudo, no estudo realizado por Teixeira et al. (2006), foram separados os efeitos de cada uma dessas dimensões do bebedouro na preferência das vacas, ficando claro que a área do espelho d'água é quem influencia mais a escolha e consumo de água desses animais. A preferência das vacas no primeiro teste deste estudo tem respaldo nessa conclusão, uma vez que o bebedouro preferido pelas vacas foi o de maior espelho d'água. Todavia, a ausência de uma preferência entre os bebedouros $125 \mathrm{C}$ e $100 \mathrm{R}$ pode ser explicada pelo fato desses dois bebedouros possuírem uma mistura de diferentes características preferidas pelos bovinos. O bebedouro 100R, além de ter maior espelho d'água, era mais baixo. Teixeira et al. (2006) encontraram, por parte dos animais, uma tendência preferencial por bebedouros mais baixos, quando as demais condições eram iguais. Por outro lado, o bebedouro $125 \mathrm{C}$, apesar de ser mais alto e ter menor espelho d'água, era redondo, e Coimbra et al. (2009) mostrou que bovinos preferem e bebem mais água de bebedouros redondos do que retangulares.

Quando tinham acesso apenas a um dos bebedouros durante o período de $24 \mathrm{~h}$, vacas leiteiras beberam mais água no bebedouro que elas haviam escolhido no teste de preferência (Machado Filho et al., 2004), foi confirmado em um estudo realizado em condições diferentes, com novilhas de corte (Coimbra et al., 2005). A disponibilização de água no bebedouro seguindo a escolha dos animais levou a um aumento no ganho de peso de novilhos de corte (Bica, 2005). Da mesma forma, a oferta de água no bebedouro de preferência por parte das vacas, possivelmente, deve favorecer a produção leiteira. A ingestão de água é fundamental para a produção leiteira, visto que, para cada litro de leite produzido, uma vaca necessita ingerir aproximadamente 3 a 5 litros de água (NRC, 2001). Portanto, espera-se que a oferta de água no bebedouro $500 \mathrm{C}$, preferido pelas vacas, propicie um maior consumo a campo em relação a outros tipos de bebedouros e aguadas naturais, favorecendo a produtividade leiteira.

Em longo prazo, aspectos importantes como a subotimização do desenho dos bebedouros podem refletir-se no rendimento da produção leiteira ou mesmo no bemestar desses animais (Pinheiro Machado, 2004; Fraser, 2008). Além das questões discutidas acima, o fator competição deve ser considerado nas recomendações do desenho dos comedouros e bebedouros (Buskirk et al., 2003), principalmente porque bovinos são animais gregários, que formam uma hierarquia social dentro do grupo (Lindberg, 2001). A redução do espaço disponível para cada animal frente ao bebedouro resulta no aumento de interações agonísticas e pode limitar o acesso de alguns animais a este recurso (Albright, 1993). Além disso, a água deve estar disponível durante todo o dia, pois quando os animais têm acesso restrito à água, as vacas dominantes bebem mais que as subordinadas (Andersson e Lindgren, 1987; Hötzel et al., 2009) e as vacas lactentes bebem mais que as vacas secas e novilhas (Hötzel et al., 2009).

Os bebedouros menos preferidos neste experimento pelas vacas são muito utilizados em propriedades leiteiras da região sul do País, devido à sua disponibilidade e baixo custo. Entretanto, considerando que bovinos consomem mais água de bebedouros de sua preferência (Machado Filho et al., 2004; Coimbra et al., 2005), e que o aumento da ingestão de água favorece a produtividade leiteira (Senn et al., 1996; Burgos et al., 2001; NRC, 2001), conclui-se que o bebedouro 500C, ou outro com características semelhantes, possa ser recomendado, após a observação da preferência apresentada pelos animais durante a pesquisa.

\section{Agradecimentos}

Paula Araújo Dias Coimbra, Gisele Pereira Pacheco de Souza, Robson Munari, Gabriela Schenato Bica e estudantes e funcionários do Colégio Agrícola de Camboriú que colaboraram na coleta de dados. 


\section{Referências}

Albright, J. 1993. Feeding behaviour of dairy cattle. Journal of Dairy Science, 76 (2): 485-498.

Andersson, M.; Lindgren, K. 1987. Effects of restricted access to drinking-water at feeding, and social rank, on performance and behavior of tied-up dairy-cows. Swedish Journal of Agricultural Research, 17 (2): 77-83.

Andersson, M.; Schaar, J.; Wiktorsson, H. 1984. Effects of drinking water flow rates and social rank on performance and drinking behaviour of tied-up dairy cows. Livestock Production Science, 11: 599-610.

Bagshaw, C. S.; Thorrold, B.; Davison, M.; Duncan, I. J. H.; Matthews, L. R. 2008. The influence of season and of providing a water trough on stream use by beef cattle grazing hill-country in New Zealand. Applied Animal Behaviour Science, 109 (2-4): 155-166.

Bica, G. S. 2005. Bebedouros: Bem-estar animal e proteção ambiental no suprimento de água para bovinos de corte. Dissertação de Mestrado, Universidade Federal de Santa Catarina, Brasil, 104pp.

Burgos, M.; Senn, M.; Sutter, F.; Kreuzer, M.; Langhans, W. 2001. Effect of water restriction on feeding and metabolism in dairy cows. American Journal of Physiology. Regulatory, Integrative and Comparative Physiology, 280: R418-R427.

Buskirk, D. D.; Zanella, A. J.; Harrigan, T. M.; Van Lente, J. L.; Gnagey, L. M.; Kaercher, M. J. 2003. Large round bale feeder design affects hay utilization and beef cow behavior. Journal of Animal Science, 81: 109-115.

Coimbra, P. A. D.; Machado Filho, L. C. P.; Nunes, P. A.; Hötzel, M. J.; Lenzi, A.; Cecato, U. 2009. Effect of water trough type on the drinking behaviour of pasture-raised beef heifers. Animal (no prelo).

Coimbra, P. A. D.; Nunes, P. A.; Machado Filho, L. C. P.; Hötzel, M. J. 2007. Water trough location, availability of shade and drinking behaviour of cattle on pasture. Proceedings of 41 Congress of the International Society for Applied Ethology, Merida, México, p.130.

Fraser, D. 2008. Toward a global perspective on farm animal welfare. Applied Animal Behaviour Science, 113 (4): 330-339.

Hötzel, M. J.; Machado Filho, L. C. P.; Teixeira, D. L.; Coimbra, P. A. D.; dos Santos, J. M. 2009. A hierarquia social e o regime de oferta influenciam o consumo de água em bovinos leiteiros. Revista Brasileira de Etologia (no prelo).
Kamphues, J. 2000. Water requirement of food producing and companion animals. Deutsche Tierarztliche Wochenschrift, 107 (8): 297-302

Lindberg, A. C. 2001. Group life. In: Keeling, L. J. \& Gonyou, H. W. (Eds.). Social behaviour in farm animals. CABI Publishing, Oxon, UK, p.37-58.

Machado Filho, L. C. P.; Teixeira, D. L.; Weary, D. M.; Von Keyserlingk, M. A. G.; Hötzel, M. J. 2004. Designing better water troughs: dairy cows prefer and drink more from larger troughs. Applied Animal Behaviour Science, 89: 185-193.

Miner, J. R.; Buckhouse, J. C.; Moore, J. A. 1992. Will a water through reduce the amount of time hay-fed livestock spend in the stream (and therefore improve water quality)? Rangeland, 14 (1): 35-38.

NRC. 2001. Nutrient requirements of Dairy Cattle. $7^{\text {th }}$ rev. ed. National Academy Press, Washington, USA, 242pp.

Pinheiro Machado, L. C. 2004. Pastoreio racional voisin Tecnologia agroecológica para o terceiro milênio. Cinco Continentes, Porto Alegre, Brasil, 314pp.

Senn, M.; Gross-Luem, S.; Kaufmann, A.; Langhans, W. 1996. Effect of water deprivation on eating patterns of lactating cows fed grass and corn pellets ad lib. Physiology Behavior, 60: 14131418 .

Sheffield, R. E.; Mostaghimi S.; Vaughan D. H.; Collins E. R.; Allen V. G. 1997. Off-stream water sources for grazing cattle as a stream bank stabilization and water quality BPM. American Society of Agriculture Engineer, 40: 595-604.

Siegel, S. 1981. Estatística não-paramétrica (para ciências do comportamento). McGraw-Hill, São Paulo, Brasil, 350pp.

Snedecor, G. W.; Cochran, W. G. 1989. Statistical Methods. $8^{\text {th }}$ ed. Iowa State University Press, Ames, USA, 503pp.

Teixeira, D. L.; Hötzel, M. J.; Machado Filho, L. C. 2006. Designing better water troughs 2 . Surface area and height, but not depth, influence dairy cows' preference. Applied Animal Behaviour Science, 96: 169-175.

Willms, W.; Kenzie, O. R.; Mcallister, T. A.; Colwell, D.; Vieira, D.; Wilmshurst, J. F.; Entz, T.; Olson, M. E. 2002. Effects of water quality on cattle performance. Journal of Range Management, 55 (5): 452-460 